\title{
Total Internal Reflection Fluorescence Microscopy to Study Microtubule Dynamics
}

\author{
J.L. Ross, ${ }^{*}$ T. Hawkins, ${ }^{*}$ K. Hingorani** \\ * Department of Physics, University of Massachusetts Amherst, Amherst, MA 01003 \\ ** Molecular and Cellular Biology Program, University of Massachusetts Amherst, MA 01003
}

Microtubules are cytoskeletal filaments inside cells with essential roles for cell morphology, intracellular transport, and mitosis. The heterodimer, made from alpha-beta tubulin, is the basic subunit of the microtubule lattice. These dimers associate longitudinally to form protofilaments and laterally to create sheets that roll into a tube, typically with 13 protofilaments in vivo. Since dimers always associate in the same direction, the entire microtubule filament is polarized with alpha at the minus end and beta at the plus end. Microtubules have an intrinsic ability to polymerize (grow) and depolymerize (shrink) with stochastic switching between these two states termed catastrophes (growing to shrinking) and rescues (shrinking to growing). This process is termed "dynamic instability" and it can be recapitulated in vitro (1). In cells, microtubules also display dynamic instability, but the dynamics are finely tuned depending on the cell type and the location within the cell. MAPs are responsible for enhancing these dynamics by nucleating, promoting polymerization, stabilizing, or destabilizing microtubules (2-13).

We are interested in recapitulating the dynamics observed in cells by systematically adding stabilizing and destabilizing microtubule-associated proteins. One interesting set of microtubule stabilizing proteins is the Ending Binding (EB) proteins. EB was discovered as a protein that binds to adenomatous polyposis coli (APC) tumor suppressor protein (13). EB proteins can bind to (2) and are enriched at the tips of microtubules $(14,15)$. Plus-tip tracking has been recapitulated in vitro using the yeast homolog, Mal3 (16), and with mammalian EB proteins $(3,17)$. Both in cells and in vitro, it has been demonstrated that EB proteins bind transiently to the end and turn over rapidly.

We are also interested in the effects of destabilizing microtubule-associated proteins, such as the microtubule-severing enzymes. Microtubule severing enzymes are a novel class of MAPs that use ATP to remove tubulin subunits from the microtubule. They can remove tubulin from the middle of the microtubule, called severing. They can also remove tubulin from the ends, appearing as depolymerization (18). With these two modes of operation, microtubule-severing enzymes would have the unique ability to compete with stabilizing MAPs microtubules to control the dynamic instability of microtubules.

In order to measure the dynamics while observing the binding of associated proteins, we will use two-color total internal reflection fluorescence (TIRF) microscopy on purified system. We systematically alter the concentrations of stabilizing and destabilizing proteins to examine the effects on microtubule dynamics. We can recapitulate the dynamics of microtubules observed in cells by controlling the activity of stabilizing and destabilizing microtubule-associated proteins.

1. Mitchison, T., and Kirschner, M. (1984) Dynamic Instability of Microtubule Growth, Nature 312 , 237-242. 
2. Berrueta, L., Kraeft, S. K., Tirnauer, J. S., Schuyler, S. C., Chen, L. B., Hill, D. E., Pellman, D., and Bierer, B. E. (1998) The adenomatous polyposis coli-binding protein EB1 is associated with cytoplasmic and spindle microtubules, Proc Natl Acad Sci U S A 95, 10596-10601.

3. Bieling, P., Kandels-Lewis, S., Telley, I. A., van Dijk, J., Janke, C., and Surrey, T. (2008) CLIP170 tracks growing microtubule ends by dynamically recognizing composite EB1/tubulin-binding sites, J Cell Biol 183, 1223-1233.

4. Brouhard, G. J., Stear, J. H., Noetzel, T. L., Al-Bassam, J., Kinoshita, K., Harrison, S. C., Howard, J., and Hyman, A. A. (2008) XMAP215 is a processive microtubule polymerase, Cell 132, 79-88.

5. Grigoriev, I., Gouveia, S. M., van der Vaart, B., Demmers, J., Smyth, J. T., Honnappa, S., Splinter, D., Steinmetz, M. O., Putney, J. W., Hoogenraad, C. C., and Akhmanova, A. (2008) STIM1 is a MT-plus-end-tracking protein involved in remodeling of the ER, Curr Biol 18, 177-182.

6. Helenius, J., Brouhard, G., Kalaidzidis, Y., Diez, S., and Howard, J. (2006) The depolymerizing kinesin MCAK uses lattice diffusion to rapidly target microtubule ends, Nature 441, 115-119.

7. Komarova, Y., Lansbergen, G., Galjart, N., Grosveld, F., Borisy, G. G., and Akhmanova, A. (2005) EB1 and EB3 control CLIP dissociation from the ends of growing microtubules, Mol Biol Cell 16, 5334-5345.

8. Maiato, H., Khodjakov, A., and Rieder, C. L. (2005) Drosophila CLASP is required for the incorporation of microtubule subunits into fluxing kinetochore fibres, Nat Cell Biol 7, 42-47.

9. Manna, T., Honnappa, S., Steinmetz, M. O., and Wilson, L. (2008) Suppression of microtubule dynamic instability by the +TIP protein EB1 and its modulation by the CAP-Gly domain of p150glued, Biochemistry 47, 779-786.

10. McNally, F. J., and Vale, R. D. (1993) Identification of katanin, an ATPase that severs and disassembles stable microtubules, Cell 75, 419-429.

11. Mennella, V., Rogers, G. C., Rogers, S. L., Buster, D. W., Vale, R. D., and Sharp, D. J. (2005) Functionally distinct kinesin-13 family members cooperate to regulate microtubule dynamics during interphase, Nat Cell Biol 7, 235-245.

12. Steinmetz, M. O., and Akhmanova, A. (2008) Capturing protein tails by CAP-Gly domains, Trends Biochem Sci 33, 535-545.

13. Su, L. K., Burrell, M., Hill, D. E., Gyuris, J., Brent, R., Wiltshire, R., Trent, J., Vogelstein, B., and Kinzler, K. W. (1995) APC binds to the novel protein EB1, Cancer Res 55, 2972-2977.

14. Mimori-Kiyosue, Y., Shiina, N., and Tsukita, S. (2000) The dynamic behavior of the APCbinding protein EB1 on the distal ends of microtubules, Curr Biol 10, 865-868.

15. Morrison, E. E., Wardleworth, B. N., Askham, J. M., Markham, A. F., and Meredith, D. M. (1998) EB1, a protein which interacts with the APC tumour suppressor, is associated with the microtubule cytoskeleton throughout the cell cycle, Oncogene 17, 3471-3477.

16. Bieling, P., Laan, L., Schek, H., Munteanu, E. L., Sandblad, L., Dogterom, M., Brunner, D., and Surrey, T. (2007) Reconstitution of a microtubule plus-end tracking system in vitro, Nature 450, 1100-1105.

17. Dixit, R., Barnett, B., Lazarus, J. E., Tokito, M., Goldman, Y. E., and Holzbaur, E. L. (2009) Microtubule plus-end tracking by CLIP-170 requires EB1, Proc Natl Acad Sci U S A 106, 492-497.

18. Zhang, D., Grode, K. D., Stewman, S., Diaz, D., Liebling, E., Curie, J., Buster, D. W., Asenjo, A. B., Sosa, H. J., Ross, J., Ma, A., Rogers, S. L., and Sharp, D. J. (2011) Drosophila Katanin is a microtubule depolymerase that regulates cortical-microtubule plus-end interactions and cell migration, Nature Cell Biology accepted. 